\title{
Faceted sidewalls of silicon nanowires: Au-induced structural reconstructions and electronic properties
}

\author{
Tao Xu, ${ }^{1}$ Jean Philippe Nys, ${ }^{1}$ Ahmed Addad, ${ }^{2}$ Oleg I. Lebedev,${ }^{3}$ Ana Urbieta, ${ }^{4}$ Billel Salhi, ${ }^{1}$ Maxime Berthe, ${ }^{1}$ \\ Bruno Grandidier, ${ }^{1, *}$ and Didier Stiévenard ${ }^{1}$ \\ ${ }^{1}$ Département ISEN, Institut d'Electronique, de Microélectronique et de Nanotechnologie (IEMN), (CNRS, UMR 8520), 41 bd Vauban, \\ 59046 Lille Cedex, France \\ ${ }^{2}$ Laboratoire de Structure et Propriétés de l'Etat Solide, Université des Sciences et Technologies de Lille, 59655 Villeneuve d'Ascq, \\ France \\ ${ }^{3}$ EMAT, Physics Department, University of Antwerp, Groenenborgerlaan 171, B2020 Antwerpen, Belgium \\ ${ }^{4}$ Departamento de Fisica de Materiales, Universidad Complutense de Madrid, 28040 Madrid, Spain \\ (Received 29 January 2009; revised manuscript received 22 October 2009; published 2 March 2010)
}

\begin{abstract}
Si nanowires with a $\langle 111\rangle$ orientation, synthesized by vapor-liquid-solid process with low silane partial pressure reactant and gold as the catalyst, are known to exhibit sawtooth facets containing gold adsorbates. We report herein the study of the nanowire morphology by means of transmission electron microscopy and scanning tunneling microscopy. The nanowires consist of faceted sidewalls. The number of the sidewalls changes from 12 to 6 along the growth axis, giving rise to nanowires with an irregular hexagonal cross section at their base. The sidewalls are covered with Au-rich clusters. Their facets also exhibit atomic structures that reveal the presence of gold, resulting from the diffusion of gold during the growth. Based on these observations, the tapering of the nanowire is found to be related to two contributions: the reduction in the catalyst particle size during the growth and lateral overgrowth from the direct incorporation of $\mathrm{Si}$ species onto the nanowire sidewalls. Because the rearrangement of atoms at surfaces and interfaces might affect the growth kinetics, the trigonal symmetry as well as the higher lateral growth rate on the widest sidewalls are explained from the existence of an interfacial atomic structure with two inequivalent parts in the unit cell. Finally, spectroscopic measurements were performed on the major facets and revealed a metallic behavior at $77 \mathrm{~K}$.
\end{abstract}

DOI: 10.1103/PhysRevB.81.115403

PACS number(s): 61.46.Km, 73.22.-f, 68.37.Ef

\section{INTRODUCTION}

Metal nanoparticles are known to act as seed particles that kinetically promote the self-assembly of oriented singlecrystal semiconductor nanowires (NWs). ${ }^{1}$ While such NWs have been successfully used in numerous electronic devices, a better understanding of the growth process is still necessary, in particular, because the role of the metal particles has been found to be more complex than initially thought. ${ }^{2}$ For example, when Si NWs are synthesized from gold seed particles by the vapor-liquid-solid (VLS) growth method, the morphology of the NWs changes depending on the growth conditions. At high silane partial pressure, the nanowires have a constant diameter all along the shaft whereas at low silane partial pressure, the NWs are tapered and their length is limited. ${ }^{3}$ Such variations have been attributed to the diffusion of gold atoms from the seed particle toward the NW sidewalls during the growth. ${ }^{4-6}$

As the adsorption of metals at the surface of semiconductors causes a modification of the atomic reconstruction, ${ }^{7,8}$ similar atomic rearrangements are expected to occur on the NW sidewalls. Such modifications are related to the lowering of the surface free energy by the metallic adsorbates. A flat surface can thus be turned into a "hill and valley" structure or vice versa, depending on the orientation of the surface and the concentration of the adsorbates. Si NWs grown at low silane partial pressure show indeed sidewalls that are faceted,,${ }^{9,10}$ and such observations thus suggest that the reconstruction of the facets are related to the gold atoms. The presence of gold atoms on the NW sidewalls is also very likely to modify the electronic structure at the surface and it raises questions about the role of the surface to account for the conduction measured in the NWs. ${ }^{11,12}$

In this work, we investigate the morphology of $\langle 111\rangle$-oriented $\mathrm{Si} \mathrm{NWs}$ when the NWs are grown on a $\mathrm{Si}(111)$ surface at low silane partial pressure by Au-catalyzed chemical-vapor deposition. By combining observations of the NW sidewalls with electron microscopies and scanning tunneling microscopy (STM), we observe the existence of two regions along the growth axis of the NWs, the highest one having a dodecagonal cross section that turns into an irregular hexagonal cross section at the base of the NWs. The width of the sidewalls is dependent on the sidewall orientations and all sidewalls consist of facets. High-angle annular dark field (HAADF) scanning transmission electron microscopy (STEM) shows that the facets are covered with gold clusters, indicating the diffusion of gold from the catalyst particle. This flow of gold atoms is also supported by the characterization of the facet atomic structure, where imaging the facets at the atomic resolution clearly reveals that the facet reconstructions are different from the surface reconstructions obtained on bare Si surfaces having the same orientations. Although these results match well with a reduction in the NW cross section during the growth, the tapering is found to be mainly related to lateral growth, due to the direct incorporation of Si species onto the sidewalls. Differences in the width of the $\langle 112\rangle$ sidewalls, already below the catalyst particle and also visible when the lateral growth proceeds, favors the atomic reconstruction of the $\mathrm{Au}-\mathrm{Si}(111)$ facets and interface to affect the growth kinetics. Indeed, atomic recon- 
structions with a unit cell being divided in two inequivalent structures impose to the surface and interface atoms to overcome two different energy barriers before their incorporation into the bulk structure. Finally, probing the electronic structure of the facets, that are the most frequently observed with STM, shows that these facets are conducting around the Fermi-level position.

\section{EXPERIMENTAL DETAILS}

Si NWs were synthesized on a Si (111) substrate by the vapor-liquid-solid mechanism using the chemical-vapor deposition (CVD) technique. Au was used as catalyst and these seed particles were obtained from the controlled formation of $\mathrm{Au}$ islands on the $\mathrm{Si}(111)$ surface in ultrahigh vacuum (UHV), as described in Ref. 13. In order to obtain vertical NWs, with a [111] growth direction, the flow of the $\mathrm{SiH}_{4}$ source gas was set to a much smaller value [12 standard cubic centimeters per minute $(\mathrm{sccm})]$ than the flow rate of the carrier gas, a mixture of $\mathrm{Ar}$ and $\mathrm{H}_{2}(150 \mathrm{sccm})$, the total pressure in the chamber being set at $1.1 \mathrm{mbar}$. The growth temperature was $550{ }^{\circ} \mathrm{C}$. Figure 1 (a) shows a scanning electron microscope (SEM) image of a Si NW synthesized with these growth conditions. The NW grew in an upright position, indicating that the NW orientation is along the [111] direction. In addition to the Au catalyst particle visible at the top of the wire, the NW shaft looks tapered with faceted sidewalls.

The cleavage of the vertically oriented NWs was performed by manipulating the sample into direct contact with the substrates used to subsequently image the flat-lying NWs. These substrates correspond either to holey grids covered with a very thin carbon film for high-resolution TEM (HRTEM) images or to a fresh $\mathrm{Si}(111)-(7 \times 7)$ surface, prepared by standard procedures,${ }^{14}$ for the STM measurements. Before the cleavage of the NWs for their observation by STM, the initial sample with the upright NWs was briefly heated in UHV around $800^{\circ} \mathrm{C}$ to desorb the thin oxide layer, that is observed on the NW sidewalls in the TEM images of Figs. 1(c) and 1(d). These annealed NWs were also characterized by SEM and TEM. For example, the SEM image of Fig. 1(b) shows a NW that was heated and then cleaved onto a $\mathrm{Si}(111)-(7 \times 7)$ surface. The morphology of this heated NW looks similar to the one observed in Fig. 1(a), indicating that our heating procedure did not change the irregular hexagonal morphology of the NWs. As a rule, the TEM characterizations showed that the distribution and mean size of the facets along the NWs shaft were not modified for brief annealing temperatures below $900{ }^{\circ} \mathrm{C}$.

\section{RESULTS}

From the SEM and TEM experiments, the width of the six main sidewalls ranges into two types: three sides with small widths that alternate with three larger sides, that are labeled, respectively, $\mathrm{S}$ and $\mathrm{L}$ in Fig. 1 . The $\mathrm{S}$ side facets are found to have larger heights and lengths than the facets with $\mathrm{L}$ sides. Indeed, a S side is always facing a $\mathrm{L}$ side, as it is seen from the cross section of a NW. Thus by looking in a direction
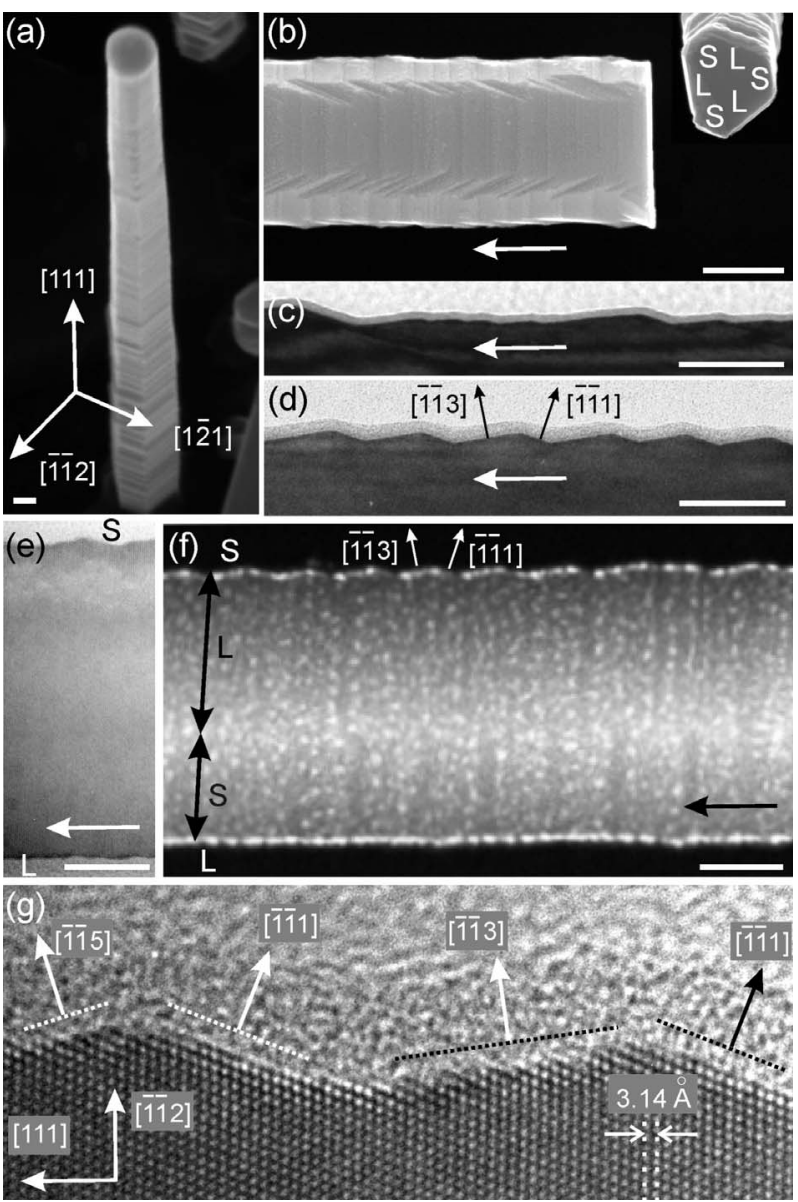

FIG. 1. (a) SEM image of a Si NW grown in the [111] direction from a gold island that was synthesized on a Si(111) surface. The orientations of two sidewalls are indicated. (b) SEM image of an annealed NW that has been cleaved and transferred onto a $\mathrm{Si}(111)$ surface. Inset: Cross section showing the NW base with an hexagonal shape. (c) TEM image of a 68-nm-diameter Si NW with irregular sawtooth-faceted sidewalls. (d) TEM image of a 300-nmdiameter Si NW with periodic sawtooth-faceted sidewalls. The facet orientations are indicated. The thin light gray layer in the TEM images corresponds to the oxide layer. (e) TEM image of a 71-nmdiameter Si NW showing a large and small corrugation for the top and bottom sidewalls, respectively. (f) HAADF STEM image of a 65-nm-diameter Si NW, where gold-rich clusters appear as bright protrusions decorating the NW sidewalls. (g) Lattice-resolved TEM image of a 75-nm-diameter Si NW. In the different images of the cleaved NWs, the horizontal arrow points toward the [111] growth direction. $\mathrm{S}$ and $\mathrm{L}$ indicate facets with small and large widths, respectively. The scale bars correspond to (a)-(d) $50 \mathrm{~nm}$ and (e) and (f) $20 \mathrm{~nm}$

normal to one of the NW edges, that are parallel to the main NW axis, a cross section of a $\mathrm{S}$ side with its opposite $\mathrm{L}$ side is obtained in the TEM and HAADF images of Figs. 1(e) and 1(f), respectively. The top sidewall ( $\mathrm{S}$ side) appears with a stronger corrugation and longer facets than the bottom sidewall (L side), where the amplitude of the sawtooth facets is much smaller [as better seen in the bottom of Fig. 1(e)], in agreement with previous observations. ${ }^{9}$

The analysis of the facet periodicity along the sidewalls shows that the NWs with the largest diameters $(>200 \mathrm{~nm})$ 
appear to have sawtooth-faceted sidewalls with a regular periodicity [Fig. 1(d)], in accordance to the results described in Ref. 10. From the knowledge of the growth direction along the [111] axis, the sidewalls are found to consist of alternating downward $\{111\}$ and upward $\{113\}$ planes. Conversely, when the diameter of the NWs is smaller, the regular periodicity of the facets is lost [Figs. 1(c), 1(e), and 1(f)]. In order to determine the facet orientation, HRTEM images along $[-110]$ zone axis were acquired. Based on the observation of the (111) atomic planes perpendicular to the NW axis seen in Fig. 1(g) for a $\mathrm{S}$ side, the direction perpendicular to this $\mathrm{S}$ side is found to be along the [112] direction. Due to the hexagonal cross section of the NWs, the directions of the other $\mathrm{S}$ sides are the $[\overline{1} 2 \overline{1}]$ and $[2 \overline{1} \overline{1}]$ directions whereas for the $\mathrm{L}$ sides, we obtain the $[11 \overline{2}],[1 \overline{2} 1]$, and $[\overline{2} 11]$ directions. In Fig. 1(g), two upward facets are clearly visible and alternate with the $\{111\}$ facets. The largest one corresponds to a $\{113\}$ plane whereas the smallest one is measured to be about $38.9^{\circ}$ off a $\{111\}$ plane, indicating that it is a $\{115\}$ facet. By performing such analysis of the facet orientations on NWs with different diameters between 50 and $200 \mathrm{~nm}$, we find that the downward facets are all oriented in a $\langle 111\rangle$ direction whereas the orientation of the upward facets might deviate from a $\langle 113\rangle$ direction.

In order to obtain the atomic structure of the different facets, scanning tunneling microscopy experiments were performed on annealed NWs with diameters ranging between 50 and $180 \mathrm{~nm}$. NWs with bigger diameters are generally difficult to image because of the limited extension of the piezoelectric ceramic supporting the tip at low temperatures. Due to the hexagonal shape of the NW shaft, we expect the NW to rest rather on a sidewall than on one of their edges. If one of their six sides is in contact with the flat $\mathrm{Si}(111)$ surface, then the opposite side is almost parallel to the scanning plane and such configuration allows a good observation of the facets. Figure 2(a) shows a large-scale STM image of a NW lying on a $\mathrm{Si}(111)-(7 \times 7)$ surface, where the facets are clearly resolved on the top sidewall of the NW. The examination of the height profile across the NW width reveals that the bottom part of the profile consists of short steep line segments on each side of the NWs. Such small line segments are attributed to the contour followed by the tip along $\mathrm{S}$ sides, as shown in the inset of Figs. 2(a) and 2(c). This configuration is also found for the NW studied in Figs. 2(b) and 2(c). Having a L side in contact with the Si surface certainly favors the most stable configuration because all the NWs that were successfully imaged with STM have a similar positioning.

Although in some cases the gold seed particle is not observed at the end of the broken NWs, the growth direction of the NWs is always obtained from measuring height profiles along the main axis; due to the tapering of the NWs, the NW diameter continuously decreases toward the [111] direction. Knowing the [111] direction then allows the identification of the downward facets, as shown in the height profile of Fig. 2(c), measured from the STM image seen in Fig. 2(b). Based on the TEM experiments described above, these facets should normally consists of $\{111\}$ planes.

Atomically resolved STM images of such facets are shown in Figs. 3(a) and 3(b) at two opposite sample volt-

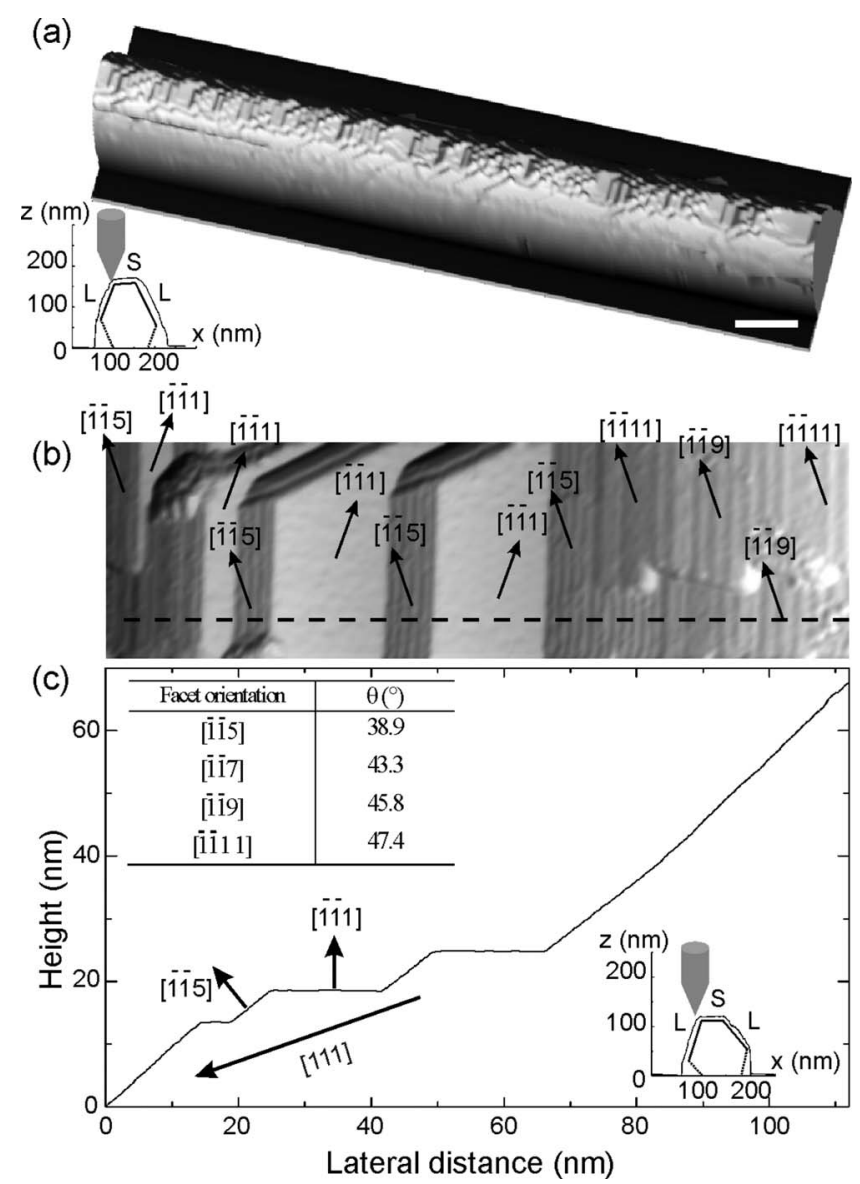

FIG. 2. (a) Large-scale three-dimensional STM image of an annealed Si NW grown along the [111] direction. Facets on the top sidewall are resolved as well as small gold-rich clusters. The average diameter of the NW is $170 \mathrm{~nm}$. The nanowire is deposited on a Si(111)- $(7 \times 7)$ surface. Scale bar: $100 \mathrm{~nm}$. Inset: Topographic height profile above a schematic transverse section of the NW. (b) STM image of a NW sidewall, where the facet orientation is indicated. The average diameter of the NW is $120 \mathrm{~nm}$. (c) Height profile measured along the dash line of the topographic image (b). Upper inset: Table giving the tilt angles $\theta$ between the [ $\overline{1} 11]$ direction and the direction of the other facets. Lower inset: Topographic height profile above a schematic transverse section of the NW shown in (b). The STM images were obtained at a sample voltage, tunneling current, and temperature of $+2.0 \mathrm{~V}, 100 \mathrm{pA}$, and $77 \mathrm{~K}$.

ages. Their atomic structure clearly differs from the wellknown $7 \times 7$ reconstruction usually observed for a bare $\mathrm{Si}(111)$ surface. Although the surface is not well ordered, some patterns are reproducibly observed and arranged in an hexagonal lattice with a sixfold periodicity with respect to the $\mathrm{Si}(111)$ substrate. Such structure is similar to the one visible in Refs. 15 and 16, and corresponds to the $6 \times 6$ phase of the $\mathrm{Au} / \mathrm{Si}(111)$ surface. The downward facets thus consist of $\{111\}$ planes in agreement with the above TEM observations. Since the $6 \times 6$ phase on the $\mathrm{Au} / \mathrm{Si}(111)$ surface occurs for $\mathrm{Au}$ coverage higher than 0.96 monolayer (ML), ${ }^{17}$ the $\{111\}$ facets roughly correspond to a Au coverage of $1 \mathrm{ML}$ (equivalent to $7.84 \times 10^{14} \mathrm{Au}$ atom $/ \mathrm{cm}^{2}$ ).

Having identified the orientation of the downward facets, the orientation of the upward facets is determined from the 


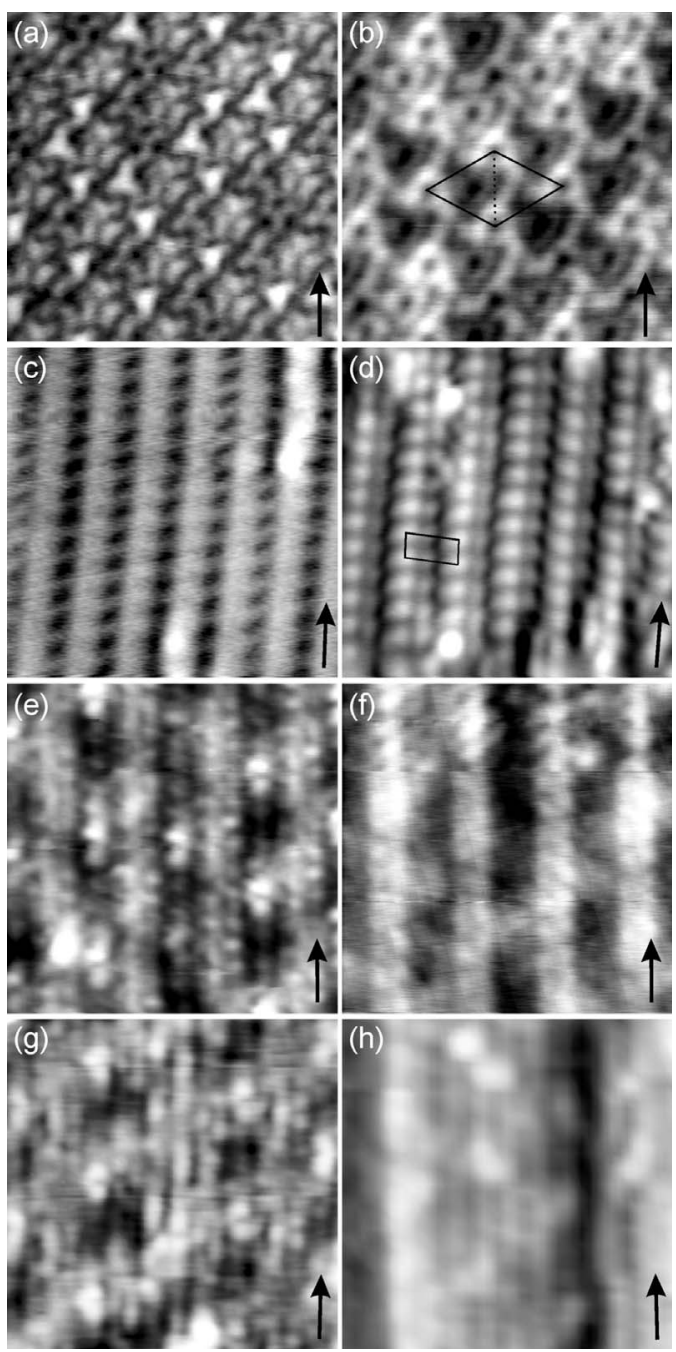

FIG. 3. Filled and empty state STM images for the (a), (b) $\operatorname{Si}\{111\},(c),(d) \operatorname{Si}\{115\}$, and (e), (f) $\operatorname{Si}\{119\}$ facets of an annealed NW. STM images for the (g) Si $\{117\}$ and (h) Si $\{1111\}$ facets. The images (a) and (b) and (e) and (f) were acquired simultaneously. The feedback parameters (sample voltage, tunneling current) were (a) $-1.3 \mathrm{~V}, 100 \mathrm{pA}$, (b) $1.3 \mathrm{~V}, 100 \mathrm{pA}$, (c) $-2.0 \mathrm{~V}, 100 \mathrm{pA}$, (d) $2.0 \mathrm{~V}$, $500 \mathrm{pA},(\mathrm{e})-2.0 \mathrm{~V}, 500 \mathrm{pA}$, (f) $2.0 \mathrm{~V}, 500 \mathrm{pA},(\mathrm{g})-1.3 \mathrm{~V}, 100 \mathrm{pA}$, and (h) $2.0 \mathrm{~V}, 100 \mathrm{pA}$. The sample temperature was $77 \mathrm{~K}$. The vertical arrow indicates a $\langle 110\rangle$ direction. The unit cells are indicated for the (a) and (b) $6 \times 6$ and (c) and (d) $4 \times 2$ reconstructions. The unit cell of the $6 \times 6$ reconstruction is divided in two inequivalent parts. All image sizes are $10 \mathrm{~nm} \times 10 \mathrm{~nm}$.

angle between the normal vector of the facet and the [1 $\overline{1} 1]$ direction with an uncertainty of $\pm 1^{\circ}$. The facet with the smallest angle corresponds to a $\{115\}$-oriented plane, as shown in the height profile of Fig. 2(c). Two other facets are also observed in Fig. 2(b) and are found to be more tilted off. A statistical analysis of the facet orientations reveals the existence of four different types of planes: the $\{115\},\{117\}$, $\{119\}$, and $\{1111\}$ planes. All these facets consist of stripes along the $\langle 110\rangle$ direction [Figs. 3(c)-3(h)]. The stripes are well resolved for the $\{115\}$ facet [Fig. 3(d)] and correspond to atomic rows yielding a $4 \times 2$ structure. In contrast, the stripes of the $\{117\}-,\{119\}$-, and $\{1111\}$-oriented facets are

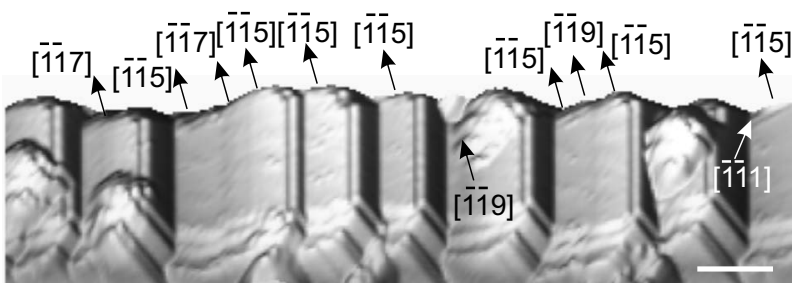

FIG. 4. Three-dimensional view of an annealed Si NW sidewall, where gold clusters are seen on the high-index planes only. The STM image was obtained at a sample voltage, tunneling current, and temperature of $-1.6 \mathrm{~V}, 100 \mathrm{pA}$, and $77 \mathrm{~K}$. Scale bar $20 \mathrm{~nm}$.

more complicated to interpret and although periodic structures are visible, it is difficult to accurately determine the reconstruction of the facets due to a high density of defects and the limited sizes of the facets [Figs. 3(e)-3(h)]. However, a comparison of the facet structures with the STM observation of a large variety of $\mathrm{Si}$ planes with orientations ranging from the [111] to the [001] clearly shows that the upward facet structures differ from the structure observed for bare $\mathrm{Si}\{115\},\{117\},\{119\}$, and $\{1111\}$ planes. $^{18}$

HAADF images, such as the one shown in Fig. 1(f), indicate that the NWs are covered with numerous bright protrusions. The HAADF, or so-called $Z$ contrast, imaging shows a strong sensitivity to the atomic number $(Z)$. This means that the heaviest atoms image as brighter dots whereas the contrast from very light atoms is negligible. Moreover, since the contrast is roughly proportional to the mean square of $Z$, even small changes in atomic structure and chemical composition can be detected. Therefore, we attribute these protrusions to Au-rich clusters in agreement with previous studies, where it was shown that the NW growth at low silane partial pressure favors the diffusion of gold from the seed particle along the NW sidewalls. ${ }^{4,5}$ Interestingly, the Au-rich clusters preferentially form on the high-index planes, as it is visible on the sawtooth facets of the top $S$ sidewall. In addition to this top and bottom sidewalls, the HAADF image of Fig. 1(f) also shows two other sidewalls, the upper one having a large (L) width whereas the lower has a small (S) width. On these sidewalls, alternating bright and dark fringes appear and are better resolved on the $S$ side, where the periodicity is known to be higher. Based on the distribution of the Au-rich clusters along the top S sidewall, such fringes reveal that the Au-rich clusters preferentially form on the high-index planes. The STM images confirm the HAADF observations, although the NWs have been annealed. For example, four gold clusters are visible in Fig. 4. All four clusters are found on the high-index planes and not on the $\{111\}$ planes, that appear black in this STM image. From (i) the presence of gold clusters on the high-index planes, (ii) the observations of atomic arrangements that do not exist on bare Si planes with similar orientations, and (iii) the existence of the $6 \times 6$ phase on the $\{111\}$ facet that is induced by $\mathrm{Au}$, we conclude that the high-index facet reconstructions are also caused by gold.

While the adsorption of metals on semiconductor surface is known to create a large variety of low-dimensional surface structures, the increased interactions and subsequent correlations that the surface electrons experience can lead to sur- 


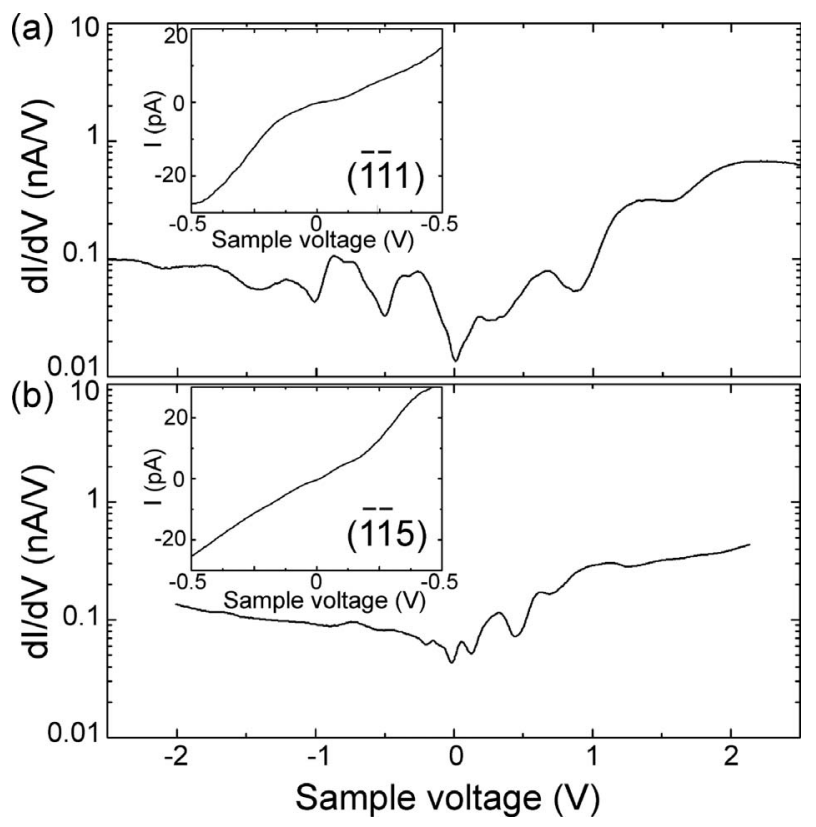

FIG. 5. Differential conductivity spectra measured on (a) $\operatorname{Si}\{111\}$ and (b) $\operatorname{Si}\{115\}$ facets of an annealed NW. The insets show the tunneling current spectra on a smaller voltage range. The feedback parameters (sample voltage, tunneling current) were (a) $-2.5 \mathrm{~V}, 100 \mathrm{pA}$ and (b) $-2.0 \mathrm{~V}, 100 \mathrm{pA}$ at a temperature of $77 \mathrm{~K}$. Both spectra were acquired with a tip-sample variable distance method where the tip was approached linearly toward the surface at a rate of 0.80 and $0.67 \AA / \mathrm{V}$, respectively.

faces with different conducting properties. ${ }^{19}$ As a result, spectroscopic measurements have been performed on the facets that are found with the highest occurrence, the $\{111\}$ and $\{115\}$ facets. In Fig. 5, the spectra measured on the $\mathrm{Au} / \mathrm{Si}\{111\}-(6 \times 6)$ and $\mathrm{Au} / \mathrm{Si}\{115\}-(4 \times 2)$ facets reveal a metallic nature of the electronic states from the $I(V)$ characteristic. Measurements of the differential conductance clearly show the existence of several bands of electronic states in the energy region around $0 \mathrm{~V}$ and extending up to $1 \mathrm{eV}$, that corresponds to the band gap of Si. For states lying below the Fermi level $(0 \mathrm{~V})$, such result is consistent with the formation of several bands of filled surface states that were probed by angle-resolved photoelectron spectroscopy for the $\mathrm{Au} / \mathrm{Si}(111)-(6 \times 6)$ (Ref. 20) and yielded, in the case of the highest band, a metallic character when the order of this phase was not perfect. ${ }^{21}$ In the case of the $\mathrm{Au} / \mathrm{Si}\{115\}-(4$ $\times 2$ ) facets, the stripes clearly correspond to atomic chains. Ideal atomic chains are known to exhibit Peierls instability with the opening of an energy gap at low temperatures. However, as the electronic wave functions decay exponentially away from the atomic chains, the electronic coupling between the chain or with the substrate can be strong enough to give a residual two-dimensional character, leaving the surface metallic, as recently found for other similar systems at low temperatures. ${ }^{22-24}$ As a result, both facets are metallic in contrast to the expected semiconductor nature of the NW core. While UHV conditions preserve the metallicity of the facets, the facet reconstructions are very much likely to be disrupted in air, when an oxide layer forms. Rather than improving the NW surface conduction, gold atoms could then act as trapping centers for free carriers. ${ }^{25}$

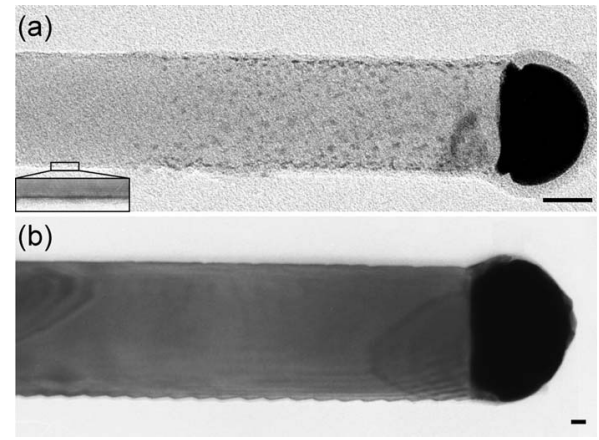

FIG. 6. (a) TEM image of a Si NW grown with a silane partial pressure of 1 mbar at a temperature of $430{ }^{\circ} \mathrm{C}$. Dark protrusions visible on the shaft below the seed particle correspond to Au-rich clusters. Inset: zoom on the NW edge to show the straight sidewall. (b) TEM image of a Si NW grown with a silane partial pressure of 0.08 mbar at a temperature of $550{ }^{\circ} \mathrm{C}$. The NW sidewalls are faceted. Scale bar: $20 \mathrm{~nm}$.

\section{DISCUSSION}

While the combined TEM and STM characterizations of the Si NWs show that their sidewalls are fully covered with gold, in agreement with a recent study, ${ }^{26}$ it is not yet clear at this point, if the gold atoms adsorbed on the NW sidewalls have any role on the NW shaft morphology or if the geometry of the facets is dictated by the Au-rich catalyst particle only. In order to determine how gold affects the NW morphology, Si NWs were grown at higher silane partial pressures and lower temperatures. Figure 6(a) shows a NW grown with a silane partial pressure of 1 mbar at a temperature of $430{ }^{\circ} \mathrm{C}$ whereas the NW seen in Fig. 6(b) was obtained with standard growth conditions (silane partial pressure of 0.08 mbar and growth temperature of $550{ }^{\circ} \mathrm{C}$ ). In contrast to this latter NW, where both types $\mathrm{L}$ and $\mathrm{S}$ of faceted sidewalls are clearly resolved, the NW of Fig. 6(a) has a different morphology. Below the gold droplet located at the top of the NW (right part of the TEM image), the shaft appears speckled over a length of $120 \mathrm{~nm}$. The dark features with sizes of a few nanometers correspond to Au-rich clusters, as it was demonstrated in Ref. 5. Such transitional region is attributed to the further growth of the NWs from residual silane gas after the interruption of the gas flow in the growth chamber and also the subsequent diffusion of gold due to the high-temperature inertia of the chamber, after silane has been fully pumped away. Alternatively, in the region below, that corresponds to constant growth conditions, the contrast on the NW shaft is homogeneous and the NW sidewalls are straight and parallel to the growth direction [inset of Fig. 6(a)]. Therefore, the silane partial pressure governs the diffusion of gold. An increase in the silane partial pressure favors the adsorption of silane molecules on the NW sidewalls while a lowering of the temperature reduces the rate of silane dissociation. As it was suggested in Ref. 5, the saturation of Si dangling bonds by silane molecules or products of the silane decomposition prevents gold from diffusing along the surface of the NW shaft. The observation of straight sidewalls implies that the diffusion of gold is crucial for the formation of faceted sidewalls. 


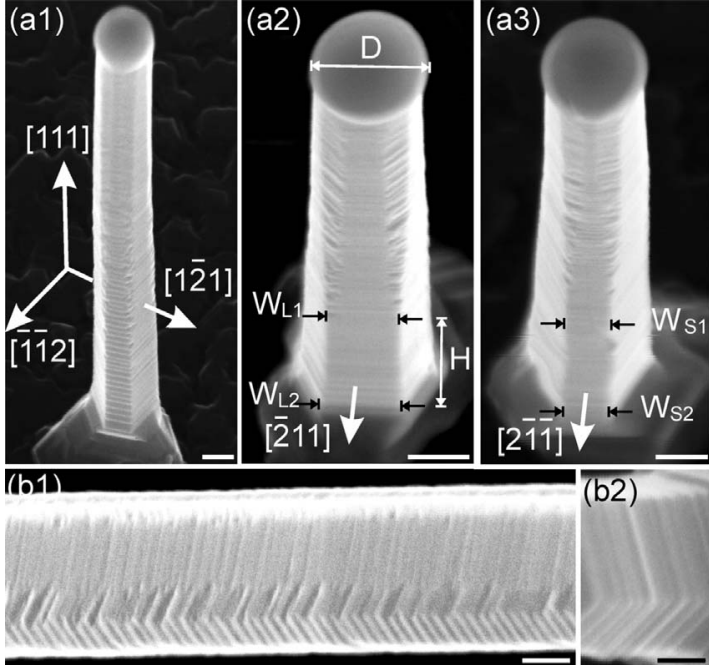

FIG. 7. (a) SEM images of a $\langle 111\rangle$-oriented Si NW viewed from different directions with the sample stage either rotated at (a1) $26^{\circ}$ or (a2) and (a3) $10^{\circ}$. The orientations of the most visible sidewalls are indicated. The diameter of the seed particle, the width of the $\mathrm{L}$ and S sidewalls at the end of the transitional region and at the NW base, and the height between the transitional region and the NW base are labeled $\mathrm{D}, \mathrm{W}_{\mathrm{L} 1} / \mathrm{W}_{\mathrm{S} 1}, \mathrm{~W}_{\mathrm{L} 2} / \mathrm{W}_{\mathrm{S} 2}$, and $\mathrm{H}$, respectively. (b) SEM images of the (b1) top and (b2) base of a $\langle 111\rangle$-oriented Si NW transferred onto a $\mathrm{Si}(111)$ surface. Scale bar: $50 \mathrm{~nm}$.

From Fig. 1, it is clear that the facets of NWs grown at a low partial silane pressure and a high temperature are found on all the six main sidewalls, conferring to the base of the NWs an irregular hexagonal cross section. In contrast, the top of the NW shown in the SEM image of Fig. 1(a) looks more fuzzy, suggesting a change in morphology during the growth. This modification cannot be related to the interruption of growth when the pressure and temperature decrease because it usually extends over several hundreds nanometers along the growth direction. Figure 7(a) shows an example of a NW, that was imaged with different view angles, and where truncated sidewalls are visible on a height of $1.9 \mu \mathrm{m}$ in addition to the six main sidewalls. The six truncated sidewalls connect the $\langle 112\rangle$-oriented sidewalls. They thus give an irregular dodecagonal cross section to the NW shaft below the catalyst particle, in agreement with results shown in Ref. 10 , where they were assigned to $\langle 110\rangle$-oriented sidewalls. These sidewalls also consist of facets and the width of the facets is seen to shrink as they are located further away from the top of the seed particle [Fig. 7(b1)]. This decrease in the truncated sidewall width takes place with a change in the facet angle with respect to the [111] direction. Such a progressive change results in an alignment of the facets for two adjacent $\mathrm{S}$ and $\mathrm{L}$ sidewalls, that become really effective, when the truncated sidewalls have disappeared [Fig. 7(b2)].

Because for short growth times, the irregular hexagonal cross section is hardly visible at the base of NWs, these observations indicate that lateral growth occurs in addition to the VLS mechanism. Therefore, the tapering of $\mathrm{Si}$ NWs grown at low silane partial pressure is partly related to the reduction in the seed particle size due to the diffusion of gold and partly to the incorporation of $\mathrm{Si}$ species impinging di-

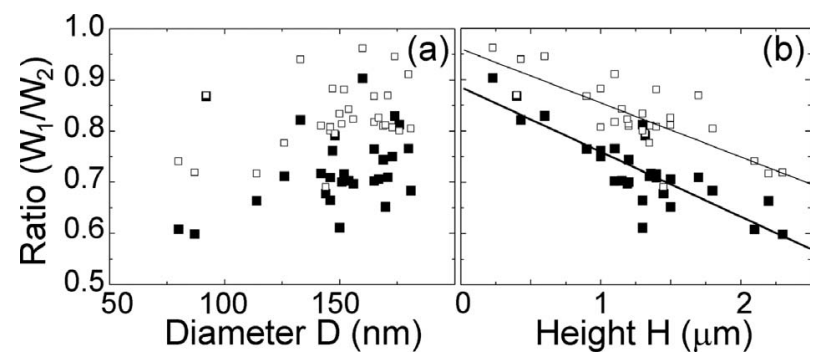

FIG. 8. Variation in the sidewall width as a function of (a) the seed particle diameter $D$ and (b) the height $H . D$ and $H$ are defined in Fig. 7. The ratios between the width at the end of the transitional region and the width at the base are plotted in filled and open squares for the L and S sidewalls, respectively.

rectly onto the sidewalls. Because the base of the Si NWs undergoes the longest exposure time to the Si species, it has the largest diameter. This latter observation is consistent with the sidewall growth obtained for III-V NWs, that is enhanced at elevated temperatures, ${ }^{27-29}$ and also for $\mathrm{Si}$ and Ge NWs grown by CVD. ${ }^{30-32}$ Considering the surface tension $\gamma$ of bare Si (111), (112), and (110) faces, it was theoretically shown that $\gamma_{111}<\gamma_{112}<\gamma_{110}$ (Ref. 33). Although the surface tensions differ when the surfaces contains gold adsorbates, such trend suggests why the $\langle 110\rangle$-oriented sidewalls disappear to the benefit of less costly $\langle 112\rangle$-oriented sidewalls, when the lateral growth proceeds.

To confirm the existence of a lateral growth, the widths of the $\mathrm{S}$ and $\mathrm{L}$ sidewalls were measured at the end of the transitional region and at the base of the NWs, in the region where the NW has an irregular hexagonal cross section. This region extend over a height $H$. By plotting the ratio between the width at the end of the transitional region and one at the base as a function of the catalyst particle diameter $D$, no direct relationship appears in Fig. 8(a). Conversely, in Fig. 8 (b), the ratio, always lower than one, is found to decrease linearly as a function of the height $H$. Since the flow of silane is constant during the growth, this result shows that the width of the facets is directly related to the duration of the growth: the facet become wider and wider as more and more Si species are directly incorporated onto the sidewalls.

Because gold is known to diffuse from the catalyst particle, leading to a reduction in particle size as the growth proceeds, the diameter of the initial particle $D_{b \text { Au }}$ can be estimated from the particle size $D$ and the total height $h$ of the NW measured by SEM after the growth. ${ }^{34}$ Considering a NW with a simplified hexagonal symmetry, as seen in Fig. 9(b), and taking into account of the small Au-rich clusters adsorbed on the sidewalls to fix the Au coverage, Fig. 9(a) clearly shows that the measured cross section $D_{b m}$ at the base of the NW is constantly larger than $D_{b A u}$. Such result confirms the overgrowth process, due to the direct incorporation of Si species onto the sidewalls. From the difference between $D_{b m}$ and $D_{b \mathrm{Au}}$, the mean lateral growth rate is found to be $1.5 \pm 0.2 \mathrm{~nm} / \mathrm{min}$. Because the mean axial growth rate is $139.6 \pm 22.8 \mathrm{~nm} / \mathrm{min}$, the lateral growth is about 90 times slower than the axial growth rate at a growth temperature of $550{ }^{\circ} \mathrm{C}$, consistent with the ratio measured in Refs. 31 and 35 . 


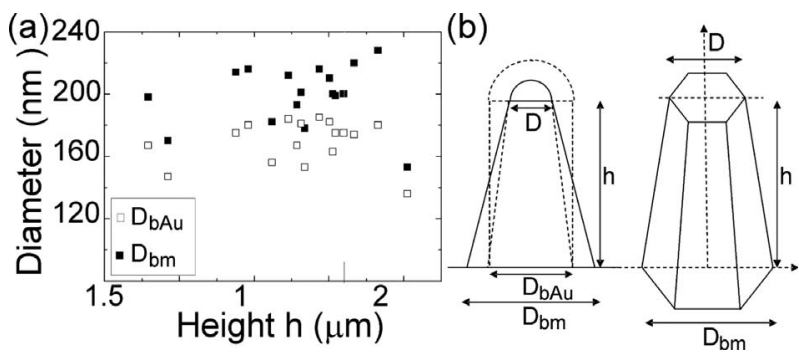

FIG. 9. (a) Measured $D_{b m}$ and estimated diameter $D_{b A u}$ at the base of the NWs, knowing their diameter $D$ and total height $h$ at the end of the growth. $D_{b \mathrm{Au}}$ corresponds to the diameter of the catalyst particle before the growth in case where there is no lateral growth and the reduction in the particle size is only caused by gold diffusion. (b) Simplified geometry of the NW to determine the variation in the diameter due to gold diffusion.

Interestingly, this overgrowth process shows a faster incorporation rate along the $\mathrm{L}$ sidewalls in comparison with the $\mathrm{S}$ sidewalls, as seen in Fig. 8(b). It is known that deposition of $\mathrm{Si}$ on a vicinal $\mathrm{Si}(111)$ surface by molecular-beam epitaxy leads to a step-flow growth pointing toward the [11 $\overline{2}]$ direction with the formation of zigzag arrays of steps perpendicular to the $[2 \overline{1} \overline{1}]$ or equivalent direction. ${ }^{36}$ Thus, the incorporation of $\mathrm{Si}$ on the NW sidewalls seems to follow a similar behavior in opposite directions. Focusing now on the top part of the NWs, where the dodecagonal cross section occurs, Fig. 7 shows that the $\mathrm{S}$ sidewalls are quite narrow in comparison with the L sidewalls. Such significant difference between the widths of the $\mathrm{S}$ and $\mathrm{L}$ sidewalls just below the catalyst particle indicates that the energy to form a sidewall perpendicular to the $[11 \overline{2}]$ direction is not equivalent to the energy required to obtain a [ $\overline{1} \overline{1} 2]$-oriented sidewall. This result closely resembles that of $\mathrm{Si}$ islands grown on the $\mathrm{Si}(111)$ surface for temperatures between 500 and $900 \mathrm{~K}$, where triangular islands are obtained rather than hexagonal islands ${ }^{37}$ and is in agreement with the shape of very large triangular Au-rich islands built on the $\mathrm{Si}(111)$ surface and observed at temperatures higher than the eutectic temperature. ${ }^{38,39}$

The occurrence of preferred growth orientations has been attributed to the influence of the surface reconstruction that is responsible for the existence of different nucleation barriers depending on the orientation of the step edges. Indeed, the $\mathrm{Si}(111)-(7 \times 7)$ dimer-adatom stacking fault surface is divided into two triangle subunits, a faulted half cell and an unfaulted half cell. Because the growth on a faulted half cell requires rearrangement of the surface atoms, the transformation of the reconstructed surface layer toward the bulk structure is associated with a larger barrier energy. Therefore, during the homoepitaxial growth of atomic terraces on the $\mathrm{Si}(111)$ surfaces, the destruction of the faulted half cells is the rate-determining process for step flow whereas the epitaxial growth on the unfaulted half cells at the lower step edge is rapid, leading to wide and narrow steps perpendicular to the $[\overline{1} \overline{1} 2]$ and $[11 \overline{2}]$ directions, respectively. ${ }^{40}$ As a result, the observation of narrow $\mathrm{S}$ and wide $\mathrm{L}$ sidewalls below the catalyst particles suggests that the growth of a Si layer at the three phase boundary between the catalyst particle and the $\mathrm{Si}$
NW is related to the existence of a unit cell with two inequivalent subunits. Such assumption would explain why the growth of the interfacial layer is easier along the steps having a $[11 \overline{2}]$ direction, implying the formation of wide steps surrounded by half unit cells with high-energy barriers. The same physical effect is expected to account for the growth of the $\mathrm{L}$ sidewalls at a higher growth rate than the $\mathrm{S}$ sidewalls below the transitional region. Looking at the reconstruction of the $\{111\}$ facets observed on the Si NW sidewalls by STM, the $6 \times 6$ phase consists of a unit cell with two different contrasts in agreement with previous STM and theoretical studies, ${ }^{41,42}$ and could be at the origin of preferential growth directions. However, it is not yet clear if this phase survives on the surface and interface of $\mathrm{Si}$ NWs covered with gold monolayer at a temperature of $550{ }^{\circ} \mathrm{C}$. Indeed, this phase is usually replaced by a $\beta-\sqrt{ } 3 \times \sqrt{3}$ phase on a flat $\mathrm{Si}$ surface covered with one monolayer of gold at temperatures higher than $350{ }^{\circ} \mathrm{C}$, as demonstrated in the Appendix. Nevertheless, because the $\beta$ - $\sqrt{3} \times \sqrt{3}$ phase corresponds to a disordered 6 $\times 6$ phase $^{17}$ and consists of domain walls running in three equivalent [112]-type directions only, both phases have a structure that well comply with the trigonal symmetry of the NWs.

In addition to variations in the sidewall widths, the orientation of the upward facets are found to change along the $\mathrm{S}$ sidewalls during the growth. It has recently been shown that the adsorption of gold on a $\mathrm{Si}(112)$ surface triggers a transformation of the surface. ${ }^{43}$ Above a critical coverage, stable facets are obtained and their orientation alternates between (111) and (113) planes. From the TEM and STM analyses, it appears that some facets are more tilted than the $\langle 113\rangle$ direction. Faceting of vicinal Si (100) surfaces by gold leads to the formation of stable (119) planes but more inclined facets with respect to the [100] direction can be obtained by decreasing the temperature or increasing the Au coverage. ${ }^{8}$ As it has been demonstrated above, silicon species are incorporated on the sidewalls during the growth. An increase in the silicon concentration on the sidewalls might thus favor the transformation of (113) planes into higher-index planes. However, at the same time, gold is expected to continuously diffuse from the catalyst particle and numerous Au-rich clusters are observed on high-index facets having different orientations, indicating that the gold coverage is higher than the critical coverage to form the high-index planes. Therefore, it is difficult to believe that fluctuations of the $\mathrm{Au}$ coverage only locks the facet orientation in the case of the NW sidewalls.

The NW growth takes place at the interface between the catalyst particle and the Si shaft, and facets are already visible just below the interface, as seen in Fig. 6(b), for example. From SEM images such as the one shown in Fig. 10 (a), it is clear that the contact angle increases as the diameter of the catalyst particle decreases. In addition, SEM top views of the catalyst particle shows that the particle has a noncircular cross section [Fig. 10(c)]. The particle base accommodates to the dodecagonal cross section of the NW with an expansion and a contraction of the particle when it is. respectively, in contact with $\langle 112\rangle-(\mathrm{L}$ and $\mathrm{S})$ and $\langle 110\rangle$ (T)-oriented sidewalls. As the contact angle increases with 

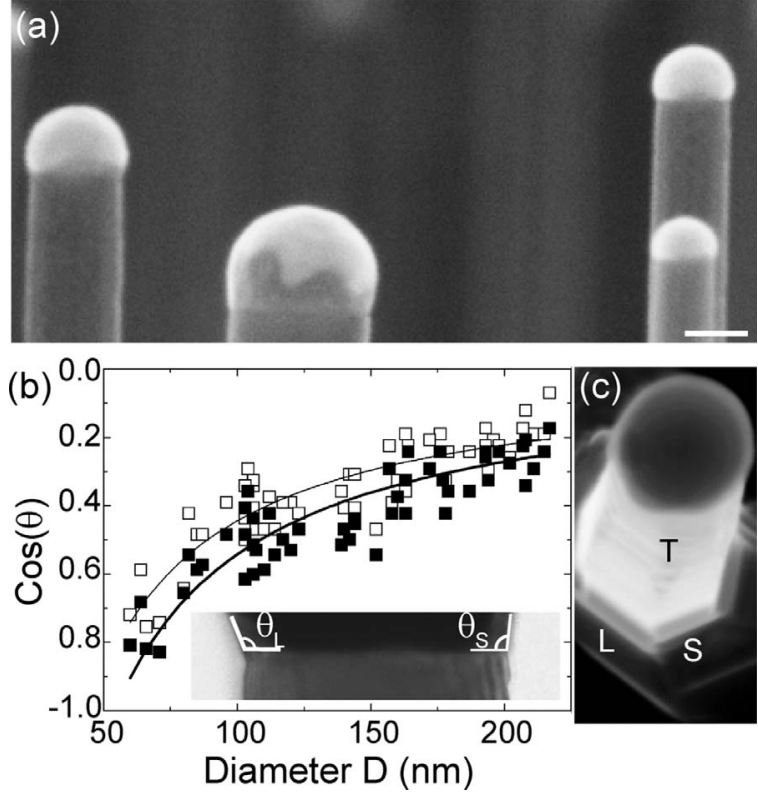

FIG. 10. (a) SEM image of seed particles located at the top of $\langle 111\rangle$-oriented Si NWs. Scale bar: $80 \mathrm{~nm}$. (b) Variation in the contact angle as a function of the catalyst particle for the $\mathrm{L}$ and $\mathrm{S}$ sidewalls. Inset: Enlarged view of the interfacial region between the top of the Si NW and the catalyst particle. (c) Tilted SEM image of a $\langle 111\rangle$-oriented Si NW, where the deformation of the particle to wet the different types of sidewalls is visible. The long, short, and truncated sidewalls are labeled $\mathrm{L}, \mathrm{S}$, and T, respectively.

smaller diameters, the deformation of the particle becomes more complex. Therefore, for NW with small diameters, the accommodation process to wet the entire top of the shaft at the three phase boundary may induce small variations in the contact angle and thus of the facet tilt.

Taking $\alpha$ as the facet angle between the NW growth direction and the direction parallel to the facet, the contact angle $\theta$ relates to $\alpha$ by the Young's equation,

$$
\cos \theta=\frac{\gamma_{s}}{\gamma_{l}} \sin \alpha-\frac{\gamma_{s l}}{\gamma_{l}}-\frac{2 \tau}{\gamma_{l}} \frac{1}{D}
$$

where $\gamma_{s}, \gamma_{s l}, \gamma_{1}$, and $\tau$ are the solid (silicon), solid-liquid interface (silicon-particle), liquid (particle), and line tensions. By measuring the contact angles of different NWs for the $S$ and $L$ sidewalls, it appears that the cosine of both contact angles $\theta_{\mathrm{S}}$ and $\theta_{\mathrm{L}}$ follows the same behavior; the angles increase as the NW gets thinner; $\theta$ being always smaller at the interface between a S sidewall and the particle in comparison with the opposite sidewall [see, for example, the inset of Fig. 10(b)]. Since the TEM and STM analyses show the existence of higher-index facets than the $\{113\}$ plane on the S sidewalls of the thin NWs, $\sin \alpha$ should also increase with decreasing diameters. If the contribution of the tilted facet is high in the Young's equation, then $\cos \theta$ is expected to increase. But the measurements of the contact angles indicate that the cosines function of the contact angle decreases for smaller and smaller diameters. Therefore, the contribution of the facet inclination to the contact angle is small and due to the limited resolution of the SEM, it is difficult to get enough accuracy to determine the interplay between the contact angle and the facet angle. Conversely, the line tension seems to govern the behavior of the contact angle with respect to the NW diameter. Indeed, the graph shown in Fig. 10(b) indicates that $\cos \theta$ varies as the inverse of the NW diameter whatever the type of the sidewall. From the fit of both types of data, the line tension can thus be measured and yields a positive value of $2.0 \pm 0.1$ $\times 10^{-8} \mathrm{~J} \mathrm{~m}^{-1}$, taking the particle surface tension as $\gamma_{1}$ $=0.85 \mathrm{~J} \mathrm{~m}^{-2} .{ }^{44}$ Such value of the line tension is in the range of line tensions commonly measured for other systems. ${ }^{45}$

While the gold coverage is higher than the one required to form the high-index planes, this study also shows that the clusters preferentially nucleate on the high-index facets rather than on the (111) facets. Such result is in agreement with the observation of Au islands formed on facet areas in the case of Au adsorption on vicinal Si (100) surfaces $^{8}$ or at the top of step bunches on vicinal $\mathrm{Si}(111)$ surfaces rather than in the middle of flat terraces. ${ }^{46}$ The atomic reconstruction of Si surfaces is dictated by the reduction in the energy through the minimization of the number of dangling bonds. In addition to dimers, high-index $\mathrm{Si}$ surfaces generally consist of rebonded steps, nonrebonded steps, and $\pi$-bonded chains. ${ }^{18}$ Because, the clusters contain not only gold but also silicon, the incorporation of the $\mathrm{Si}$ atoms is thus easier from steps and should preferentially occur on high-index facets. Indeed, the atom chains of one-dimensional surface structure on silicon, as those observed in Fig. 3, are known to originate from $\mathrm{Si}$ atoms with broken bonds, ${ }^{19}$ and it requires less energy to detach such weakly bonded atoms than $\mathrm{Si}$ atoms residing in a $\{111\}$ plane. In addition, rebonded steps introduce tensile surface stress. Similarly, the periodic linear atomic rows observed on the high-index planes in Fig. 3, are likely to induce a stress perpendicular to the $\langle 110\rangle$ direction, which modifies the interfacial area between the clusters and the facets. A decrease in the energy in the interfacial area would favor the nucleation of the islands on high-index facets rather than on a $\{111\}$ plane.

Finally, it is surprising that the STM experiments do not reveal the formation of $\{113\}$ facets on the $\mathrm{S}$ sidewall, in contrast to the TEM observations. While we cannot attribute the change in facet orientation to the annealing in UHV since $\{113\}$ facets are found for annealed NWs that are observed with TEM, the disappearance of the $\{113\}$ facets seems to be related to the removal of the oxide layer. The existence of $\mathrm{Au}-\mathrm{Si}(113)$ planes was demonstrated by adsorbing $\mathrm{Au}$ on high-index $\operatorname{Si}(5512)$ surfaces at temperatures between 600 and $800{ }^{\circ} \mathrm{C}^{47}$ Surprisingly, in this case, the (113) planes did not show any ordered reconstruction in contrast to all other high-index planes. Due to the small size of the NW sidewalls and the high probability to encounter Au clusters on the high-index planes, that certainly modify the strain in the layer, the formation of $\{113\}$ facets may be energetically precluded, when the oxide layer is removed.

\section{CONCLUSION}

In summary, by combining TEM, SEM, and STM observations of $\mathrm{Si}$ NWs grown on a $\mathrm{Si}(111)$ surface at low silane 
partial pressure, we can draw the following conclusions. First, the shape of the $\langle 111\rangle$-oriented NW cross section is dependent on the surface reconstruction that is induced by gold and requires a unit cell with two inequivalent parts. The STM topographic images point toward the $6 \times 6$ phase or the counterpart $\beta-\sqrt{ } 3 \times \sqrt{3}$ phase to account for the formation of NWs with a trigonal symmetry. Then, in addition to the tapering effect attributed to a reduction in the catalyst particle size during the growth because of gold diffusion, lateral growth has been demonstrated, turning the irregular dodecagonal cross section of the NW below the catalyst particle into an irregular hexagonal cross section at its base. Finally, the atomic structures of the facets observed on the S sidewalls have been found to be induced by gold since they differ from the reconstructions usually observed for highindex bare Si planes. Assuming a similar faceting on the $\mathrm{L}$ sidewalls, at least below the transitional region, the spectroscopic measurements indicate that the major facets are conducting even at $77 \mathrm{~K}$, conferring a metallic character to the NW sidewalls. Because faceted sidewalls increase the roughness of the NW surface and efficiently scatter surface phonons, if the periodicity of the facet orientation is lost, metallic sidewalls should be helpful to increase the figure of merit of Si NWs in thermoelectric applications. The formation of Au-rich clusters on the facets should affect in a similar manner the phonon propagation, but, these clusters, due to their self-organization on high-index facets, could also offer a natural way to control the subsequent growth of branches on the NW sidewalls.

\section{ACKNOWLEDGMENTS}

We thank C. Boyaval, Y. Coffinier, and P. Lefebvre for their technical assistance. The authors acknowledge financial support from the French National Research Agency under project PREAANS and NANOBIODETECTEUR, the Delegation Generale pour l'Armement and the European Union under the Framework 6 program, contract for an Integrated
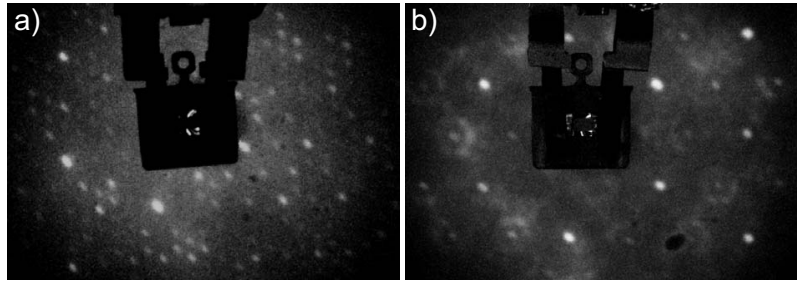

FIG. 11. (a) LEED pattern of the $\mathrm{Au} / \mathrm{Si}(111)-(6 \times 6)$ surface observed at $350{ }^{\circ} \mathrm{C}$ with a primary energy of $100 \mathrm{eV}$. (b) LEED pattern of the $\mathrm{Au} / \mathrm{Si}(111)-\beta(\sqrt{3} \times \sqrt{3})$ surface observed at $550{ }^{\circ} \mathrm{C}$ with a primary energy of $100 \mathrm{eV}$.

Infrastructure Initiative (Reference No. 026019 ESTEEM).

\section{APPENDIX}

Due to the discrepancies that exist regarding the surface phase diagram of the $\mathrm{Au} / \mathrm{Si}(111)$ surface, ${ }^{48-50}$ the surface reconstruction has been studied for a gold coverage of 1.0-1.2 $\mathrm{ML}$ as a function of the surface temperature. A $(7 \times 7)$ reconstructed $n$-doped $\mathrm{Si}(111)$ substrates was prepared by standard procedures in an UHV system with a base pressure of $10^{-10}$ Torr. Au was evaporated from a tungsten (W) filament, the Si surface being heated at temperatures higher than $360{ }^{\circ} \mathrm{C}$. The Au deposition rate was calibrated from the phase transformation of the surface. Depending on the cooling rate, either a $\beta-\sqrt{ } 3 \times \sqrt{ } 3$ phase (fast cooling) or a $6 \times 6$ phase (slow cooling) was observed by low-energy electron diffraction (LEED), consistent with Ref. 17. Starting with a $6 \times 6$ phase at room temperature, the surface was then heated. As shown in Fig. 11(a), the $6 \times 6$ phase is still visible at a temperature of $350{ }^{\circ} \mathrm{C}$. By further increasing the temperature of the sample, the $6 \times 6$ phase is replaced by a $\beta-\sqrt{ } 3 \times \sqrt{3}$ phase, in particular, at a temperature of $550{ }^{\circ} \mathrm{C}$, that corresponds to the growth temperature of the Si NWs [see Fig. 11(b)]. *bruno.grandidier@isen.iemn.univ-lille1.fr

${ }^{1}$ R. S. Wagner, in Whisker Technology, edited by A. P. Levitt (Wiley, New York, 1970).

${ }^{2}$ M. T. Borgström, G. Immink, B. Ketelaars, R. Algra, and E. P. A. M. Bakkers, Nat. Nanotechnol. 2, 541 (2007).

${ }^{3}$ A. Lugstein, M. Steinmair, Y. J. Hyun, G. Hauer, P. Pongratz, and E. Bertagnolli, Nano Lett. 8, 2310 (2008).

${ }^{4}$ J. B. Hannon, S. Kodambaka, F. Ross, and R. M. Tromp, Nature (London) 440, 69 (2006).

${ }^{5}$ M. den Hertog, J.-L. Rouvière, F. Dhalluin, P. J. Desré, P. Gentile, P. Ferret, F. Oehler, and T. Baron, Nano Lett. 8, 1544 (2008).

${ }^{6}$ T. Kawashima, T. Mizutani, T. Nakagawa, H. Torii, T. Saitoh, K. Komori, and M. Fujii, Nano Lett. 8, 362 (2008).

${ }^{7}$ S. Hasegawa, X. Tong, S. Takeda, N. Sato, and T. Nagao, Prog. Surf. Sci. 60, 89 (1999).

${ }^{8}$ H. Minoda, K. Yagi, F.-J. Meyer zu Heringdorf, A. Meier, D.
Kähler, and M. Horn von Hoegen, Phys. Rev. B 59, 2363 (1999).

${ }^{9}$ F. M. Ross, J. Tersoff, and M. C. Reuter, Phys. Rev. Lett. 95, 146104 (2005).

${ }^{10}$ T. David, D. Buttard, T. Schülli, F. Dallhuin, and P. Gentile, Surf. Sci. 602, 2675 (2008).

${ }^{11}$ J. Bauer, F. Fleischer, O. Breitenstein, L. Schubert, P. Werner, U. Gösele, and M. Zacharias, Appl. Phys. Lett. 90, 012105 (2007).

${ }^{12}$ J. E. Allen, E. R. Hemesath, D. E. Perea, J. L. Lensch-Falk, Z. Y. Li, F. Yin, M. H. Gass, P. Wang, A. L. Bleloch, R. E. Palmer, and L. J. Lauhon, Nat. Nanotechnol. 3, 168 (2008).

${ }^{13}$ T. Xu, J. P. Nys, B. Grandidier, D. Stiévenard, Y. Coffinier, R. Boukherroub, R. Larde, E. Cadel, and P. Pareige, J. Vac. Sci. Technol. B 26, 1960 (2008).

${ }^{14}$ M. Dubois, L. Perdigão, C. Delerue, G. Allan, B. Grandidier, D. Deresmes, and D. Stiévenard, Phys. Rev. B 71, 165322 (2005).

${ }^{15}$ J. Nogami, A. A. Baski, and C. F. Quate, Phys. Rev. Lett. 65, 
1611 (1990).

${ }^{16}$ S. Yamazaki, I. Matsuda, H. Okino, H. Morikawa, and S. Hasegawa, e-J. Surf. Sci. Nanotech. 3, 497 (2005).

${ }^{17}$ T. Nagao, S. Hasegawa, K. Tsuchie, S. Ino, C. Voges, G. Klos, H. Pfnür, and M. Henzler, Phys. Rev. B 57, 10100 (1998).

${ }^{18}$ A. A. Baski, S. C. Erwin, and L. J. Whitman, Surf. Sci. 392, 69 (1997).

${ }^{19}$ J. N. Crain and F. J. Himpsel, Appl. Phys. A 82, 431 (2006).

${ }^{20}$ H. M. Zhang, T. Balasubramanian, and R. I. G. Uhrberg, Phys. Rev. B 66, 165402 (2002).

${ }^{21}$ T. Okuda, H. Daimon, S. Suga, Y. Tezuka, and S. Ino, Appl. Surf. Sci. 121-122, 89 (1997).

${ }^{22}$ C. Tegenkamp, T. Ohta, J. L. McChesney, H. Dil, E. Rotenberg, H. Pfnür, and K. Horn, Phys. Rev. Lett. 100, 076802 (2008).

${ }^{23}$ A. van Houselt, T. Gnielka, J. M. J. Aan de Brugh, N. Oncel, D. Kockmann, R. Heid, K.-P. Bohnen, B. Poelsema, and H. J. W. Zandvliet, Surf. Sci. 602, 1731 (2008).

${ }^{24}$ J. A. Lipton-Duffin, A. G. Mark, J. M. MacLeod, and A. B. McLean, Phys. Rev. B 77, 125419 (2008).

${ }^{25}$ D. V. Lang, H. G. Grimmeiss, E. Meijer, and M. Jaros, Phys. Rev. B 22, 3917 (1980).

${ }^{26}$ A. Bailly, O. Renault, N. Barrett, L. F. Zagonel, P. Gentile, N. Pauc, F. Dhalluin, T. Baron, A. Chabli, J. C. Cezar, and N. B. Brookes, Nano Lett. 8, 3709 (2008).

${ }^{27}$ M. A. Verheijen, G. Immink, T. De Smet, M. T. Borgström, and E. P. A. M. Bakkers, J. Am. Chem. Soc. 128, 1353 (2006).

${ }^{28}$ K. Hiruma, K. Haraguchi, M. Yazawa, Y. Madokoro, and T. Katsuyama, Nanotechnology 17, S369 (2006).

${ }^{29}$ J. Zou, M. Paladugu, H. Wang, G. J. Auchterlonie, Y.-N. Guo, Y. Kim, Q. Gao, H. J. Joyce, H. Hoe Tan, and C. Jagadish, Small 3, 389 (2007).

${ }^{30}$ P. Aella, S. Ingole, W. T. Petuskey, and S. T. Picraux, Adv. Mater. (Weinheim, Ger.) 19, 2603 (2007).

${ }^{31}$ H. Schmid, M. T. Björk, J. Knoch, H. Riel, W. Riess, P. Rice, and T. Topuria, J. Appl. Phys. 103, 024304 (2008).
${ }^{32}$ H. Jagannathan, M. Deal, Y. Nishi, J. Woodruff, C. Chidsey, and P. C. McIntyre, J. Appl. Phys. 100, 024318 (2006).

${ }^{33}$ J.-M. Zhang, F. Ma, K.-W. Xu, and X.-T. Xin, Surf. Interface Anal. 35, 805 (2003).

${ }^{34} D_{b \mathrm{Au}}$ verified the following equation: $\frac{\pi}{18}\left(D_{b \mathrm{Au}}^{3}-D^{3}\right)=a \theta\left(D_{b \mathrm{Au}}\right.$ $+D) \sqrt{\frac{3}{16}\left(D_{b \mathrm{Au}}-D\right)^{2}+h^{2}}$, where $a^{3}$ is the atomic volume of $\mathrm{Au}$ $(a=0.26 \mathrm{~nm})$ and $\theta$ is the Au coverage on the sidewall. Based on synchrotron measurements of Si NWs grown with similar growth conditions (see Ref. 26), $\theta$ was set to $1.8 \mathrm{ML}$.

${ }^{35}$ S. Kodambaka, J. Tersoff, M. C. Reuter, and F. M. Ross, Phys. Rev. Lett. 96, 096105 (2006).

${ }^{36}$ H. Omi and T. Ogino, Thin Solid Films 380, 15 (2000).

${ }^{37}$ B. Voigtländer and T. Weber, Phys. Rev. Lett. 77, 3861 (1996).

${ }^{38}$ W. Święch, E. Bauer, and M. Mundschau, Surf. Sci. 253, 283 (1991).

${ }^{39}$ B. Ressel, K. C. Prince, S. Heun, and Y. Homma, J. Appl. Phys. 93, 3886 (2003)

${ }^{40}$ W. Shimada and H. Tochibara, Surf. Sci. 311, 107 (1994).

${ }^{41}$ M. Jałochowski, Prog. Surf. Sci. 74, 97 (2003).

${ }^{42}$ D. Grozea, E. Landree, L. D. Marks, R. Feidenhans'1, M. Nielsen, and R. L. Johnson, Surf. Sci. 418, 32 (1998).

${ }^{43}$ C. Wiethoff, F. M. Ross, M. Copel, M. Horn-von Hoegen, and F.-J. Meyer zu Heringdorf, Nano Lett. 8, 3065 (2008).

${ }^{44}$ V. Schmidt, S. Senz, and U. Gösele, Nano Lett. 5, 931 (2005).

${ }^{45}$ R. Lipowsky, P. Lenz, and P. S. Swain, Colloids Surf., A 161, 3 (2000).

${ }^{46}$ A. V. Latyshev, D. A. Nasimov, V. N. Savenko, and A. L. Aseev, Thin Solid Films 367, 142 (2000).

${ }^{47}$ J. W. Dickinson, J. C. Moore, and A. A. Baski, Surf. Sci. 561, 193 (2004)

${ }^{48}$ G. Le Lay, Surf. Sci. 132, 169 (1983).

${ }^{49}$ R. Plass and L. D. Marks, Surf. Sci. 380, 497 (1997).

${ }^{50}$ D. Grozea, E. Bengu, and L. D. Marks, Surf. Sci. 461, 23 (2000). 